OAl-PMH: http://www.indteca.com/ojs/index.php/Revista Scientific/oai

Artículo Original / Original Article

\title{
Eficiencia del Clima Organizacional y Desempeño Laboral del Instituto de Educación Superior Tecnológico del Ejército
}

\author{
Autor: Jorge Alberto Chanca Guerra \\ Universidad Nacional Mayor de San Marcos, UNMSM \\ jorge.chancag@gmail.com \\ Lima, Perú \\ https://orcid.org/0000-0002-9285-7743
}

\section{Resumen}

Es importante lograr las relaciones significativas entre el personal y la organización, para alcanzar que el clima organizacional incida favorablemente sobre el desempeño laboral. La presente investigación plantea como objetivo establecer el nivel de relación entre la eficiencia del clima organizacional con el desempeño laboral del personal militar del Instituto de Educación Superior Tecnológico Público del Ejército del Perú (IESTPE) - 2020. La investigación del estudio fue aplicada, ubicada en un enfoque de investigación básica, y en un nivel descriptivo-correlacional. El diseño es no experimental. Y que como unidad de análisis propone la eficiencia del clima organizacional y el desempeño del personal militar. La muestra se representó por 120 estudiantes del III año de las diversas carreras profesionales del IESTPE a quienes se les designó una encuesta. Los resultados indican que existe relación de $r=, 631$ " entre las variables en la muestra. Y que concluye que existe relación significativa entre las variables eficiencia del clima organizacional y la variable desempeño laboral en el Instituto de Educación Superior Tecnológico Público del Ejército, reconociendo que el clima organizacional es un factor determinante y fundamental dentro de las organizaciones, haciendo que el personal perciba el entorno que lo rodea de manera positiva o negativa.

Palabras clave: eficiencia; clima organizacional; desempeño laboral; relaciones humanas; toma de decisiones.

Código de clasificación internacional: 6109.99 - Otras (Clima Organizacional).

\footnotetext{
Cómo citar este artículo:

Chanca, J. (2021). Eficiencia del Clima Organizacional y Desempeño Laboral del Instituto de Educación Superior Tecnológico del Ejército. Revista Scientific, 6(22), 270-290, e-ISSN: 25422987. Recuperado de: https://doi.org/10.29394/Scientific.issn.2542-2987.2021.6.22.14.270-290
}

Fecha de Recepción: 30-07-2021
Fecha de Aceptación: 13-10-2021
Fecha de Publicación: 05-11-2021 
OAI-PMH: http://www.indteca.com/ojs/index.php/Revista Scientific/oai

Artículo Original / Original Article

\title{
Efficiency of the Organizational Climate and Work Performance of the Institute of Technological Higher Education of the Army
}

\begin{abstract}
It is important to achieve meaningful relationships between staff and the organization, to ensure that the organizational climate has a favorable impact on job performance. The objective of this research is to establish the level of relationship between the efficiency of the organizational climate with the work performance of the military personnel of the Institute of Higher Public Technological Education of the Peruvian Army (IESTPE) - 2020. The study research was applied, located in a basic research approach, and at a descriptive-correlational level. The design is non-experimental. And that as a unit of analysis proposes the efficiency of the organizational climate and the performance of military personnel. The sample was represented by 120 students of the third year of the various professional careers of the IESTPE who were assigned a survey. The results indicate that there is a relationship of $r=.631$ " between the variables in the sample. And that concludes that there is a significant relationship between the efficiency variables of the organizational climate and the job performance variable in the Public Technological Institute of Higher Education of the Army, recognizing that the organizational climate is a determining and fundamental factor within organizations, making staff perceive the environment around you in a positive or negative way.
\end{abstract}

Keywords: efficiency; organizational climate; job performance; human relations; decision making.

International classification code: 6109.99 - Other (Organizational climate).

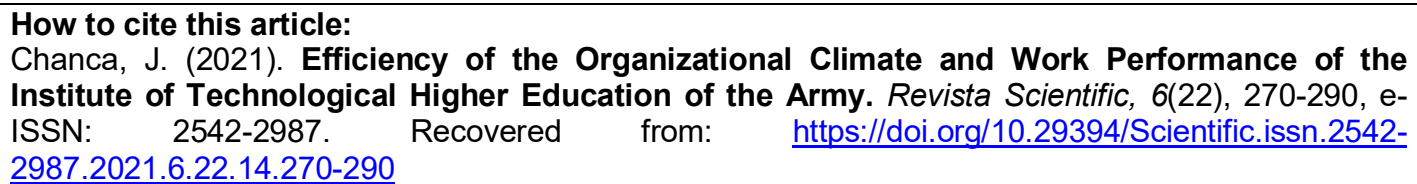

Date Received:

30-07-2021
Date Acceptance:

13-10-2021
Date Publication:

05-11-2021 


\section{Introducción}

El presente estudio tiene como objetivo establecer el nivel de relación entre la Eficiencia del clima organizacional con el desempeño laboral del personal militar. Dentro de las Fuerzas Armadas del País del Perú especialmente en la Escuela Técnica del Ejército (ETE), fundada el 13 de mayo del año 1974, otorgándole su funcionamiento a partir del año 1975; de esta manera, en el 2011 con Resolución de la Comandancia General del Ejército Nº 0475 emitida por la Dirección de Planeamiento (DIPLAN) del 18 de Julio del mismo año, se aprobó el cambio de denominación de la "Escuela Técnica del Ejército" por la de Instituto de Educación Superior Tecnológico del Ejército (IESTPE) "Sargento 2do Fernando Lores Tenazoa".

Teniendo como misión formar a los futuros Sub Oficiales del Ejército del Perú, y donde no escapa a esta realidad, ya que por ser una institución militar de organización vertical, se fundamenta en una estructura jerárquica piramidal, centrando la responsabilidad en la cima de la misma, donde todo orden militar, supone una manifestación de voluntad dirigida de modo imperativo por el superior jerárquico al subordinado; por ende, no se ejerce un buen trato al personal militar, además, no existe un tipo estudio sobre el clima organizacional en el IESTPE.

La cual motivan la insatisfacción laboral, la baja proactividad, pérdida del sentido de pertenencia hacia la institución, entre otros; en la actualidad, el IESTPE, no cuenta con instrumentos para la medición del clima organizacional, por tanto consideran que hacerlo no es importante para una institución militar y que no aportara nada a la gestión de los directivos como en los resultados; sin conocer que el clima organizacional puede ser un nexo u obstáculo para el buen desempeño dentro de la organización, siendo un elemento de influencia en el comportamiento del personal militar.

Además, dichos miembros realizan su labor por un tema de presión "orden militar", más no por una convicción propia como profesionales de la 
guerra en el ámbito educativo. Esta realidad se ha visto marcada e influida también por la presencia de COVID-19 en el mundo, haciendo que se manifiesten cambios acelerados y continuos, para evitar los comportamientos no adecuados y se manifieste el estrés ante la presencia de la pandemia en la actuación de los instructores militares dentro de la organización; es decir, que las relaciones laborales y de organización no se vean afectadas o afecten el clima laboral y consecuentemente el desempeño de quienes laboran en ella.

\subsection{Análisis teórico relevante para la investigación}

\subsubsection{Clima organizacional}

Es necesario considerar el sustento teórico en el abordaje del problema de la investigación, es así donde se hace referencia al clima organizacional, que es el ambiente real entre los miembros de la organización; por ello Chiavenato (2009a), nos señala que:

El clima organizacional es la calidad o la suma de las características ambientales percibidas o experimentadas por los miembros de la organización, e influye poderosamente en su comportamiento. El concepto del clima organizacional abarca una amplia gama de factores ambientales que influyen en la motivación. Se refiere a las propiedades motivacionales del ambiente de la organización, es decir, aquellos aspectos de la organización que provocan distintas clases de motivación en sus miembros (pág. 261).

El concepto destaca la importancia de las diferencias individuales, colaboraciones grupales, interacciones sociales y participación de los empleados para lograr un clima organizacional adecuado, que se logra como producto de las prácticas de liderazgo, las prácticas de comunicación y las características duraderas y sistemáticas de la relación de trabajo entre el personal y los departamentos de cualquier organización.

En este sentido, Colque (2011a): en su trabajo de investigación tuvo como objetivo "determinar la relación que existe entre las características 
predominantes del clima organizacional del comando de educación y doctrina del ejército y el nivel de actuación en operaciones psicológicas del personal de oficiales, recientemente egresado" (pág. 11).

Esta disertación se trazó como fin establecer cómo se relacionan los rasgos más prominentes del ecosistema laboral del personal militar de oficiales especializados del Ejercito del Perú. Según Contreras (2018a), tiene como resultado que:

[...] Existe una relación significativa $(p=0,000)$ en el nivel de correlación positiva considerable $(r=0,756)$ entre el clima organizacional y el desempeño laboral. Finalmente, las conclusiones indican que existe relación significativa entre el clima organizacional y el desempeño laboral del personal civil y militar del Cuartel General de la Tercera Brigada de Caballería "Tacna", periodo 2016, con un nivel de significancia de 0,05 y Rho de Spearman $=0,756$ y p-valor $=0,000<0,05$. (pág. 10).

La meta de la disertación fue establecer un vínculo de dos variables: $\mathrm{X}=$ ecosistema laboral y $\mathrm{Y}=$ performance de trabajo de los trabajadores civiles y de la milicia del Cuartel General de la Tercera Brigada de Caballería en Tacna. Acorde con esto, Rico y Sánchez (2014), concluye que:

Los resultados obtenidos en la medición del clima organizacional de Unidades Militares-Batallón de Infantería de Marina No 12, se obtuvo un puntaje total de 16799 de 18900 puntos posibles, lo cual arroja un nivel de cumplimiento del 88.8\% que corresponde a una categoría "Excepcional" (pág. 13).

El llamado ecosistema de laburo se presenta como un tópico de suma fundamental y relevancia a los ojos de las instituciones militares, para la esfera productiva o servicial, ya que esto está relacionado con los requerimientos sobre satisfacer los fines trazados por la organización. 


\subsubsection{Clima y cultura organizacionales}

Al respecto, Louffat (2018), nos menciona que el clima y cultura son elementos importantes y necesario de comprender en la administración del potencial humano y que hace mención que:

[...] Constituyen al mismo tiempo dos conceptos fundamentales: el del clima y la cultura organizacionales; esto, porque ambos refieren al comportamiento de una organización, es decir, como actúan, sienten y piensan los trabajadores de manera individual, y como actúan, sienten y piensan los trabajadores de manera individual, y como actúan, sienten y piensan en sus relaciones con los colegas de la empresa. Para conocer el clima organizacional se realizan mediciones periódicas, las cuales podrían ser consideradas como 'fotografías', por lo estático del momento que se capta (pág. 215).

Al respecto hace énfasis sobre el ecosistema y lo cultural y que son puntos fundamentales y constituyentes de urgencia con el fin de entender sobre el potencial de desarrollo del personal con el fin de que su percepción sea de forma positiva con relación a la empresa y sus colegas.

\subsubsection{Clima organizacional y la satisfacción laboral desde la percepción del capital humano}

El clima organizacional y la satisfacción laboral se considera relevante dentro de las organizaciones, así como la gestión del capital humano como un aspecto intangible que implica un componente importante en el logro de los objetivos. En esa línea, Pedraza (2018), señala que:

Los empleados, finalmente, representan ese capital intangible en forma de conocimientos, experiencias, habilidades, destrezas, valores y capacidades que pertenecen a los individuos, quienes, con su potencial, hacen posible que las organizaciones logren sus resultados empresariales, de allí la relevancia de ver a los trabajadores como un capital valioso. (pág. 91). 
Este marco teórico es de mucha importancia, porque toma mucha atención a cómo manejar el "capital humano", entendido como un factor imprescindible que no puede ser tocado, es abstracto, pero que implica una fuerza importante para la capacitación de los trabajadores, nivel de instrucción, etc. Y que tendrá una incidencia en cómo uno logra sus finalidades.

\subsubsection{La evaluación del desempeño}

La evaluación del desempeño sirve como herramienta de gestión que se utiliza para comprobar el nivel de cumplimiento de los objetivos trazados a nivel particular o grupal. Por ello, Chiavenato (2007), define que:

Es una apreciación sistemática de cómo cada persona se desempeña en un puesto y de su potencial de desarrollo futuro. Toda evaluación es un proceso para estimular o juzgar el valor, la excelencia y las cualidades de una persona. La evaluación de los individuos que desempeñan papeles dentro de una organización se hace aplicando varios procedimientos que se conocen por distintos nombres, como evaluación el desempeño, evaluación de méritos, evaluación de los empleados, informes de avance, evaluación de la eficiencia en las funciones, etc. (pág. 243).

Testear el performance de laburo es fundamental para medir qué tanto se satisfacen las metas estipuladas desde un principio. La evaluación del desempeño sirve como herramienta de gestión que se utiliza para comprobar el nivel de cumplimiento de los objetivos trazados a nivel particular o grupal

\subsubsection{Práctica pedagógica en el Ejército, Caso: Colombia}

En referencia al tema, Albornoz, Fernández y Betancur (2020), nos mencionan la investigación y sistematización de la práctica pedagógica de los docentes de las escuelas de formación y capacitación militar, de acuerdo con los procesos de transformación en estos últimos tiempos, lo que demuestra que: 
OAl-PMH: http://www.indteca.com/ojs/index.php/Revista Scientific/oai

Artículo Original / Original Article

La presente obra, "Práctica pedagógica en el Ejército Nacional de Colombia. Reflexiones en torno a la formación, gestión educativa y la investigación" explora los avances educativos de algunas escuelas de formación y capacitación del Ejército Nacional de Colombia, enfatizando la importancia del conocimiento científico, el saber pedagógico, la instrucción y el rol del maestro en el sistema de educación de la Fuerza (pág. 9).

La labor investigativa y la praxis de los maestros formativos y que ejercen instrucción de la fuerza en la milicia, teniendo en consideración los cambios constantes y el dinamismo de los últimos años.

\subsubsection{Formación y desarrollo}

Señalando a Dolan, valle, Jackson y Schuler (2007a): el perfeccionamiento como el progreso de los recursos humanos, además en el contexto de una perspectiva estratégica, permite preparar las capacidades necesarias para lograr los objetivos previstos, también nos señalan que:

La formación y el desarrollo del empleado consisten en un conjunto de actividades cuyo propósito es mejorar su rendimiento presente o futuro, aumentando su capacidad a través de la modificación y potenciación de sus conocimientos, habilidades y actitudes. Aunque la formación es frecuentemente usada como desarrollo, ambos términos no son sinónimos. La formación trata de proporcionar al empleado habilidades específicas o corregir deficiencias en su rendimiento. Por el contrario, el desarrollo hace referencia al esfuerzo de la organización para proporcionar a los empleados las habilidades que necesitará en el futuro (pág. 167).

El entrenamiento y la evolución del trabajador hace, gracias al entrenamiento y capacitación con tal de potenciar su performance no solo en el hoy sino también en el mañana, otorgando una habilidad más fina haciendo más fuerte lo cognoscitivo, lo habilidoso y lo actitudinal. 


\section{Metodología (Materiales y métodos)}

La investigación fue de tipo aplicada, el diseño es no experimental, acorde con esto, Kerlinger y Lee (2002), nos afirman que:

La investigación no experimental es la búsqueda empírica y sistemática en la que el científico no posee control directo de las variables independientes, debido a que sus manifestaciones ya han ocurrido o a que son inherentemente no manipulables. Se hacen inferencias sobre las relaciones entre las variables, sin intervención directa, de la variación concomitante de las variables independiente y dependiente (pág. 504).

De lo que los autores, se interpreta que el diseño es "no experimental" pues se dejará intacta la "variable independiente". De igual forma, nuestra indagación es "transversal" ya que la información es obtenida en una fecha específica y la finalidad es detallar lo que varía y examinar la influencia.

La muestra se seleccionó por conveniencia, según Arias-Gómez, Villasís-Keever y Miranda (2016): "consiste en la selección por métodos no aleatorios de una muestra cuyas características sean similares a las de la población objetivo. También puede ser que el investigador seleccione directa e intencionadamente los individuos de la población" (pág. 201). Por lo tanto, la metodología de muestreo no probabilístico por conveniencia, es la elección libre que pueda optar el investigador para el desarrollo del trabajo de científico.

La población fue conformada por 200 estudiantes que pertenecen al tercer año del Instituto de Educación Superior Tecnológico Público del Ejercito (IESTPE), para lo cual se consideró para este presente estudio el método de muestreo no probabilístico por conveniencia, en la cual fueron seleccionados 120 alumnos de las diferentes carreras profesionales del instituto. Asimismo, mencionados alumnos se encargaron de evaluar la eficiencia del clima organizacional y el desempeño laboral del personal militar, variables que conforman para este presente estudio a través de la técnica de recolección de 
datos (encuesta), e instrumentos (cuestionarios).

\section{Resultados}

Se analizó y se procesó la información con Excel, procesos de tabulación, tablas de interpretación y análisis de resultados. A fin de determinar los porcentajes obtenidos en las encuetas realizadas a los alumnos del instituto militar, clasificándolas dentro de un contexto analítico acompañado con las herramientas estadística adecuada, plasmados en los datos recolectados y que a continuación se detalla.

Tabla 1. Resultados de variable eficiencia del clima organizacional (cuestionario aplicado a los alumnos).

\begin{tabular}{|l|c|c|c|c|}
\hline \multicolumn{1}{|c|}{ Descripción } & F & \% & \% Val. & \% Acum. \\
\hline Deficiente & 60 & 33,3 & 33,3 & 33,3 \\
Regular & 45 & 41,7 & 41,7 & 75,0 \\
Bueno & 15 & 25,0 & 25,0 & 100,0 \\
\hline Total & 120 & 100,0 & 100,0 & \multicolumn{1}{|c}{} \\
\cline { 1 - 3 } & &
\end{tabular}

Fuente: El Autor (2020).

En la tabla 1, se interpreta que un total de $41,7 \%$ de la muestra indica que el clima organizacional es percibido de manera regular, mientras que consideran que es deficiente un promedio de $33,3 \%$ y que tiene un nivel bueno en un $25,0 \%$.

Tabla 2. Resultados de la dimensión nivel de comunicación (cuestionario aplicado a los alumnos).

\begin{tabular}{|l|c|c|c|c|}
\hline Descripción & F & \% & \% Val. & \% Acum. \\
\hline Deficiente & 18 & 26,7 & 26,7 & 26,7 \\
Regular & 78 & 43,3 & 43,3 & 70,0 \\
Bueno & 24 & 30,0 & 30,0 & 100,0 \\
\hline Total & 120 & 100,0 & 100,0 & \multicolumn{1}{|c}{} \\
\cline { 1 - 3 } & & &
\end{tabular}

Fuente: El Autor (2020).

Se aprecia en la tabla 2, que un total de $43,3 \%$ de los entrevistados indicaron que respecto a la dimensión nivel de comunicación consideran que 
es regular, mientras un $26,7 \%$ es deficiente y solamente el $30,0 \%$ señalan que es de buen nivel.

Tabla 3. Resultados de la dimensión relaciones humanas (cuestionario aplicado a los alumnos).

\begin{tabular}{|l|c|c|c|c|}
\hline Descripción & F & \% & \% Val. & \% Acum. \\
\hline Deficiente & 21 & 28,3 & 28,3 & 28,3 \\
Regular & 72 & 56,7 & 56,7 & 85,0 \\
Bueno & 27 & 15,0 & 15,0 & 100,0 \\
\hline Total & 120 & 100,0 & 100,0 & \\
\end{tabular}

Fuente: El Autor (2020).

Se distingue en la tabla 3 , que un total de $56,7 \%$ de los entrevistados indicaron que respecto a la dimensión relaciones humanas consideran que es regular, mientras que el $28,3 \%$ es deficiente y solo el $15 \%$ señalan que es de bueno.

Tabla 4. Resultados de la dimensión pertinencia en la toma de decisiones (cuestionario aplicado a los alumnos).

\begin{tabular}{|l|c|c|c|c|}
\hline Descripción & F & \% & \% Val. & \% Acum. \\
\hline Deficiente & 24 & 30,0 & 30,0 & 30,0 \\
Regular & 75 & 41,7 & 41,7 & 71,7 \\
Bueno & 21 & 28,3 & 28,3 & 100,0 \\
\hline Total & 120 & 100,0 & 100,0 & \\
\hline
\end{tabular}

Fuente: El Autor (2020).

Respecto a la toma de decisiones, en la tabla 4, se tiene claro según los resultados logrados que un total de $41,7 \%$ de los entrevistados manifiestan que es regular mientras que el $30,0 \%$ reconoce que es deficiente y $28,3 \%$ indica que es bueno.

Tabla 5. Resultados de la variable desempeño laboral del personal militar (cuestionario aplicado a los alumnos).

\begin{tabular}{|l|c|c|c|c|}
\hline \multicolumn{1}{|c|}{ Descripción } & F & \% & \% Val. & \% Acum. \\
\hline Malo & 21 & 21,7 & 21,7 & 21,7 \\
Regular & 84 & 63,3 & 63,3 & 85,0 \\
Bueno & 15 & 15,0 & 15,0 & 100,0 \\
\hline Total & 120 & 100,0 & 100,0 & \multicolumn{1}{|c}{} \\
\cline { 1 - 3 } & & &
\end{tabular}

Fuente: El Autor (2020). 
Respecto a la variable desempeño laboral, los resultados expuestos en la tabla 5 , indican que un $63,3 \%$ del total menciona que el desempeño laboral es regular, $21,7 \%$ manifiestan que es malo y solo el $15,0 \%$ reconocen que el nivel es bueno.

\subsection{A partir del resultado obtenido se hace la contrastación de la} hipótesis general

\subsubsection{Hipótesis general}

La Eficiencia del Clima Organizacional se podría relacionar directamente con el desempeño laboral del personal militar del Instituto de Educación Superior Tecnológico Público del Ejército del Perú - 2020.

\subsubsection{Hipótesis nula}

La Eficiencia del Clima Organizacional no se podría relacionar directamente con el desempeño laboral del personal militar del Instituto de Educación Superior Tecnológico Público del Ejército del Perú - 2020.

Nivel de significancia de confianza: $95 \%(\alpha=0.05)$

Regla de decisión: Si r valor $<0,05$; se refuta la hipótesis nula.

Si r valor $\geq 0,05$; no se refuta la hipótesis nula.

Tabla 6. Resultados de las variables Eficiencia del clima organizacional y el desempeño laboral (Coeficiente de correlación de Spearman).

\begin{tabular}{|l|l|l|c|c|}
\hline \multicolumn{2}{|l}{} & $\begin{array}{l}\text { Eficiencia del Clima } \\
\text { organizacional }\end{array}$ & $\begin{array}{c}\text { Desempeñ } \\
\text { o Laboral }\end{array}$ \\
\hline $\begin{array}{l}\text { Rho de } \\
\text { Spearman }\end{array}$ & Eficiencia del & $\begin{array}{l}\text { Coeficiente de } \\
\text { correlación }\end{array}$ & 1,000 &, $631 "$ \\
& Clima & - &, 000 \\
& organizacional & Sig. (bilateral) & 40 & 120 \\
\hline & Desempeño & Coeficiente de &, $631 ”$ & 1,000 \\
& Laboral & correlación &, 000 & - \\
& Sig. (Bilateral) & 120 & 120 \\
\hline
\end{tabular}

Fuente: El Autor (2020). 
Como se expone en la tabla 6, se aplicó el Coeficiente de Correlación Spearman entre las variables eficiencia del clima organizacional y la variable desempeño laboral del personal militar del Instituto de Educación Superior Tecnológico Publico del Ejercito, existiendo una correlación de $r=, 631$ " en la muestra.

Figura 1. Resultados de la Relación entre las variables (representada en la Campana de Gauss).

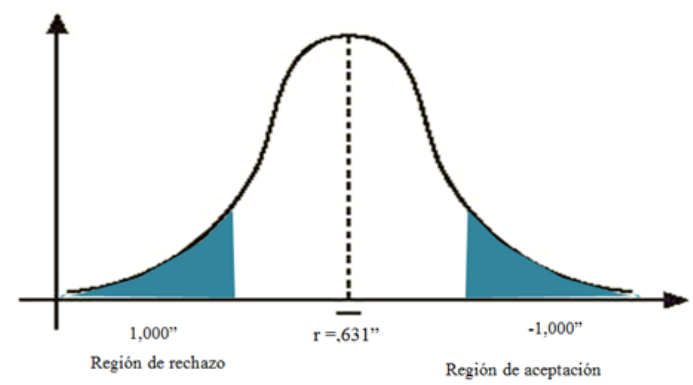

Fuente: El Autor (2020). 
Como se expone en la figura 2, se usó el Coeficiente de Correlación Spearman para confrontar lo hipotético entre la dimensión nivel de comunicación y la variable desempeño laboral del personal militar en el Instituto militar, arrojando la relación de $r=, 493$ " en la muestra.

Figura 3. Resultados de la dimensión relaciones humanas y el desempeño laboral (representada en la Campana de Gauss).

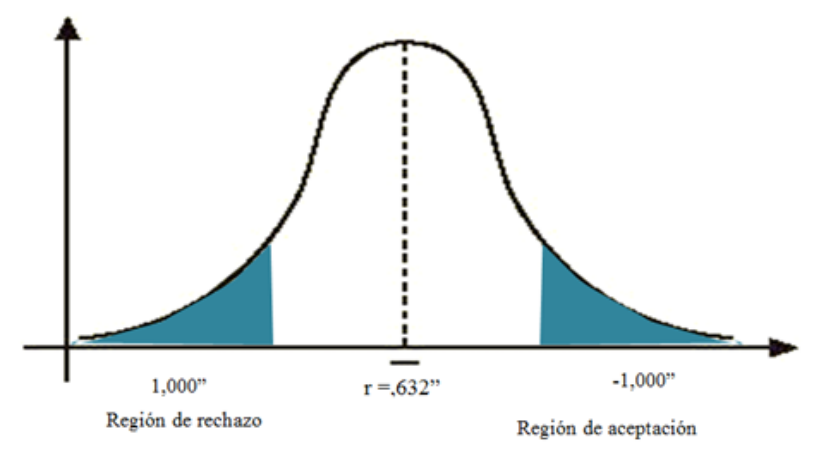

Fuente: El Autor (2020).

En la figura 3, se utilizó el Coeficiente de Correlación Spearman para confrontar lo hipotético entre la dimensión relaciones humanas y la variable desempeño laboral del personal militar en el Instituto militar, obteniendo una correlación de $=, 632$ " en la muestra.

Figura 4. Resultados de la dimensión pertinencia en la toma de decisiones y el desempeño laboral (representada en la Campana de Gauss).

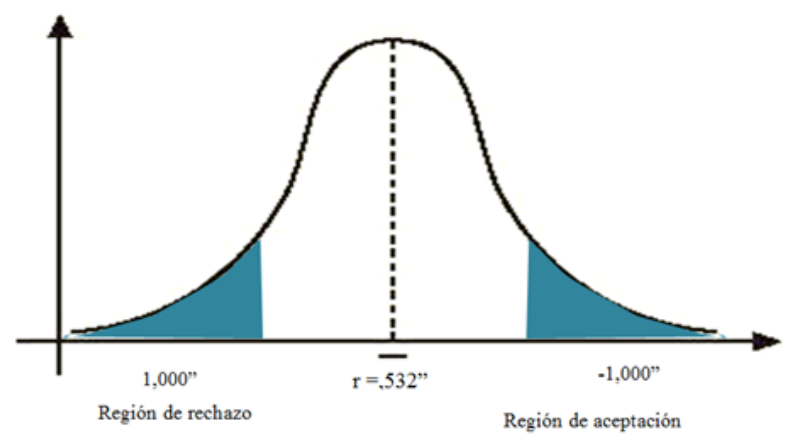

Fuente: El Autor (2020). 
Para la figura 4, existe relación de $r=, 532$ " entre la dimensión pertinencia en la toma de decisiones y la variable desempeño laboral del personal militar en la muestra. Aplicado con el Coeficiente de Correlación Spearman para confrontar lo hipotético.

\section{Discusión}

El resultado de la investigación sobre la principal hipótesis del estudio referida a despejar que la eficiencia del clima organizacional se podría relacionar directamente con el desempeño laboral del personal militar del Instituto de Educación Superior Tecnológico Público del Ejército del Perú 2020.

Para poder establecer la existencia de relación se aplicó el Rho de Spearman, que arrojo que $r=, 631$ " entre las variables eficiencia del clima organizacional y la variable desempeño laboral en la muestra, de manera similar a lo que resultó en el estudio de Contreras (2018b): que existe relación significativa entre el clima organizacional y el desempeño laboral del personal civil y militar del Cuartel General de la Tercera Brigada de Caballería de Tacna. Además del estudio de Palacios (2019), reconoce que existe una significativa relación entre las dos variables y que:

[...] Esto nos lleva a creer que el clima organizacional es un factor fundamental dentro de las organizaciones, considerando que el desempeño depende de la percepción que tenga el personal sobre el entorno que lo rodea, mismo que puede ser negativo o positivo (pág. 12).

Es importante además mencionar que el clima organizacional abarca factores ambientales diversos que influyen en la motivación y que consecuentemente provoca un clima organizacional favorable o desfavorable en el desempeño laboral dentro de la institución.

Sobre la hipótesis especifica 1 resultó que existe relación de $r=, 493$ " 
entre la dimensión nivel de comunicación y la variable desempeño laboral en la muestra, que se podría comparar al estudio de Alva (2012), en el que se:

[...] Reflejan que la Dimensión de las Relaciones Humanas son las que más influyen en que la Policía Nacional del Perú presente un clima organizacional medio, ya que, según el Criterio de Patterson, le corresponde a este Clima Organizacional Medio que el desempeño de los comisarios solo sea Eficaz, es decir, que solo cumple en el corto plazo el cumplimiento de las metas, haciendo uso racional y administrando de forma apropiada los escasos recursos con que cuenta la Comisaria (pág. 7).

Cabe mencionar que las relaciones humanas dentro de las organizaciones son las más importantes en la interacción individual o grupal que se desarrolla en un ambiente percibido de forma efectiva con el fin de cumplir con los objetivos previstos por las instituciones cívicas como policiales.

El estudio interpreta que un total de 43,3\% de los entrevistados indicaron que respecto a la dimensión nivel de comunicación consideran que es regular, mientras que la consideran deficiente en $26,7 \%$ y 30,0 indica que es de buen nivel. Sin dejar de lado que Chiavenato (2009b): además lo reconoce como la manera en que se transmite y reciben las comunicaciones dentro de la organización.

Sobre la hipótesis especifica 2 resultó que existe relación de $r=, 632 "$ entre la dimensión relaciones humanas y la variable desempeño laboral en la muestra, como se refleja en el estudio de Colque (2011b): para quien "el clima organizacional y el nivel de actuación de los oficiales especializados en operaciones psicológicas son independientes" (pág. 14). Las relaciones humanas interpersonales por decir de Chiavenato (2007b): requieren de un eficiente desarrollo y engranaje dentro de la organización, ya que solo trabajando en equipo se logra los objetivos de la institución. Es importante tener en cuenta la propuesta de Dolan, Jackson y Schuler (2007b), para quienes: 
El trabajo de planificación de los recursos humanos debe tomar en consideración a todo el conjunto de factores, entre ellos los cambios de los valores sociales. Los valores con respecto al trabajo; el estancamiento de la productividad se relaciona a menudo con el descenso o la desaparición del valor compromiso y esfuerzo en el trabajo (pág. 97).

Los valores sociales dentro de las organizaciones son fundamentales, ya que implica en todos los niveles, jerarquías y que se caracteriza desde el respeto y la identificación con el trabajo que desarrolla las personas dentro de las instituciones públicas y privadas.

Sobre la hipótesis especifica 3 resultó que existe relación de $r=, 532$ " entre la dimensión pertinencia en la toma de decisiones y la variable desempeño laboral en la muestra. Respecto a la toma de decisiones se tiene claro según los resultados logrados que un total de $41,7 \%$ de la muestra en el IESTP del Ejército manifiestan que la toma de decisiones es regular mientras que $30,0 \%$ reconoce que es deficiente y $28,3 \%$ indica que es bueno. Todo se alinea con lo indicado por Chiavenato (2009c), para quien:

La toma de decisiones es un proceso que consiste en hacer un análisis y elegir entre varias opciones un curso de acción. Toda decisión implica de seis elementos: Una persona que toma la decisión, los objetivos, las preferencias, las estrategias, la situación y el resultado (pág. 227).

En efecto para realizar la toma de decisiones se debe de optar con todas las informaciones necesarias donde implica objetivos, estrategias con el fin de decidir un rumbo y obtener los resultados esperados que fueron planificados con antelación a fin de cumplir los objetivos.

\section{Conclusiones}

Se concluye que existe correlación significativa de $r=, 631$ " entre las variables "eficiencia del clima organizacional y la variable desempeño laboral 
en el Instituto de Educación Superior Tecnológico Público del Ejército (IESTPE)". Admitiendo, que el "clima organizacional" es un elemento primordial y determinante en las organizaciones, concibiendo en el entorno personal de forma efectiva o negativa.

Se determinó la existencia de correlación significativa de $r=, 493$ " entre el indicador "nivel de comunicación" y la variable "desempeño laboral" en el IESTPE, en razón que si en la entidad existe un buen "nivel de comunicación" el desempeño de los instructores militares se incrementará de forma significativa.

Se deriva que existe correlación significativa de $r=632$ " entre las "relaciones humanas" y la segunda variable en el Instituto militar, desde el inicio se reconoce que las "relaciones humanas" casualmente admiten que se ejecuten las coordinaciones y compromisos para obtener un adecuado "desempeño laboral" dentro de la institución.

Se deduce que existe relación significativa de $r=, 532$ " entre la "pertinencia en la toma de decisiones" y la segunda variable en el instituto, se admite que le indicador en mención debe ser dinámica y no monótona; a fin de que se acepte por parte de los directivos a cargo y se sientan incluidos en la organización, para el logro de los objetivos trazados.

\section{Referencias}

Albornoz, L., Fernández, A., \& Betancur, C. (ed.). (2020). Práctica pedagógica en el Ejército Nacional de Colombia: Reflexiones en torno a la formación, gestión educativa y la investigación. Primera edición, ISBN: 978-958-52414-5-9. Bogotá, Colombia: Escuela Militar de Cadetes "General José María Córdova".

Alva, R. (2012). Relación del clima organizacional con el desempeño de los comisarios, comisarías tipo “A” de la VII DIRTEPOL - año 2012. Tesis. Perú: Universidad Nacional Mayor de San Marcos. Recuperado 
de: https://hdl.handle.net/20.500.12672/5814

Arias-Gómez, A., Villasís-Keever, M., \& Miranda, M. (2016). El protocolo de investigación III: la población de estudio. Revista Alergia México, 63(2), 201-206, e-ISSN: 0002-5151. Recuperado de: https://www.redalyc.org/articulo.oa?id=486755023011

Chiavenato, I. (2007a,b). Administración de recursos humanos: Los sistemas de administración de las organizaciones humanas. México: McGraw-Hill//nteramericana Editores, S.A.

Chiavenato, I. (2009a,b,c). Comportamiento organizacional: Clima organizacional. Monterrey, México: McGraw-Hill//nteramericana Editores, S.A.

Colque, D. (2011a,b). El clima organizacional del comando de educación y doctrina del ejército y su relación con el nivel de actuación en operaciones psicológicas en el personal de oficiales recientemente egresados de la Escuela Militar de Chorrillos. Tesis. Tacna, Perú Universidad Nacional Jorge Basadre Grohmann. Recuperado de: http://repositorio.unjbg.edu.pe/handle/UNJBG/686

Contreras, P. (2018a,b). Clima organizacional y desempeño laboral del personal civil y militar del Cuartel General de la Tercera Brigada de Caballería Tacna, 2016. Tesis. Lima, Perú: Universidad Nacional de Educación "Enrique Guzmán y Valle". Recuperado de: http://repositorio.une.edu.pe/handle/UNE/2395

Dolan, S., Valle, R., Jackson, S., \& Schuler, R. (2007a,b). La gestión de recursos humanos: Formación y desarrollo. Madrid, España: McGraw-Hill/Interamericana Editores, S.A.

Kerlinger, F., \& Lee, H. (2002). Investigación del comportamiento. Métodos de investigación en ciencias sociales. $4^{a}$ edición. México: McGrawHill.

Louffat, E. (2018). Administración del potencial humano: Clima y cultura 
organizacionales. Perú: Cengage Learning Editores, S.A.

Palacios, D. (2019). El clima organizacional y su relación con el desempeño laboral del personal administrativo de los distritos de Salud Pública de la provincia de Manabí - Ecuador. Tesis. Perú: Universidad Nacional Mayor de San Marcos. Recuperado de: https://hdl.handle.net/20.500.12672/10989

Pedraza, N. (2018). El clima organizacional y su relación con la satisfacción laboral desde la percepción del capital humano. Revista Lasallista de Investigación, 15(1), 90-101, e-ISSN: 1794-4449. Recuperado de: https://www.redalyc.org/articulo.oa?id=69559148009

Rico, J., \& Sánchez, A. (2014). Caracterización del clima organizacional en unidades militares: Caso "Batallón de Infantería de Marina $\mathrm{N}^{\circ} .12$ ". Tesis. Cartagena de Indias, Colombia: Universidad Tecnológica de Bolívar. Recuperado de:

https://repositorio.utb.edu.co/handle/20.500.12585/3680 


\section{Jorge Alberto Chanca Guerra}

e-mail: jorge.chancag@gmail.com

Nacido en Huancayo, Perú, el 25 de marzo del año 1981.

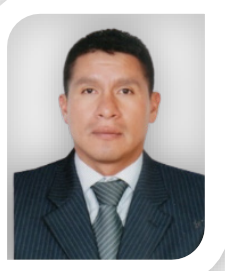

Suboficial Egresado de la Escuela Técnica del Ejército (ETE), del Perú desde el 2006; Lcdo. en Administración de Negocios Internacionales por la Universidad Alas Peruanas (UAP); Inscrito en el Colegio de Licenciados en Administración (CLAD) No 17954; y Egresado de la Maestría con mención en Gestión Pública por la Universidad Nacional Mayor de San Marcos (UNMSM), con sede en Lima, Perú, desde el año 2019; actualmente Jefe de Personal de la Dirección de Telemática y Estadística del Ejercito (DITELE), perteneciente al Cuartel General del Ejercito del Perú.

El contenido de este manuscrito se difunde bajo una Licencia de Creative Commons ReconocimientoNoComercial-Compartirlgual 4.0 Internacional 